\title{
Effects of Lattice Evolution and Ordering on the Microwave Dielectric Properties of Tin-Modified $\mathrm{Li}_{3} \mathrm{Mg}_{2} \mathrm{NbO}_{6}$-Based Ceramics
}

\author{
Xing Zhang ${ }^{1,2}$, Xiao Zhang $^{3}$, Zixuan Fang $^{1,2,{ }^{* *}}$, Zhe Xiong ${ }^{1,2}$, Hongyu \\ Yang $^{1,2}$, Shuren Zhang ${ }^{1,2}$, Bin Tang ${ }^{1,2, *}$
}

1 State Key Laboratory of Electronic Thin Films and Integrated Devices, University of Electronic Science and Technology of China, Chengdu, 610054, China

2 National Engineering Research Center of Electromagnetic Radiation Control Materials, University of Electronic Science and Technology of China, Chengdu, 610054, China

3 China Key System \& Integrated Circuit Co., Ltd. Wuxi, 214035, China

*Corresponding author: tangbin@uestc.edu.cn
** Corresponding author: zixuanfang@uestc.edu.cn

Figure and Table Caption Lists:

Table S1 Bond length $\left(\mathrm{d}_{\mathrm{ij}}\right)$, bond valence parameters $\left(\mathrm{R}_{\mathrm{ij}}\right)$, the valence of chemical bonds $\left(\mathrm{v}_{\mathrm{ij}}\right)$, the observed valence of atoms $\left(\mathrm{V}_{\mathrm{ij}}\right.$ ), and global instability index (GII) of $\mathrm{Li}_{3} \mathrm{Mg}_{2-x / 3} \mathrm{Sn}_{x} \mathrm{Nb}_{1-2 x / 3} \mathrm{O}_{6}$ $(0 \leq x \leq 1.1)$ ceramics sintered at $1290^{\circ} \mathrm{C}$ for $4 \mathrm{~h}$.

Fig. S1 XPS core-level (a-d) Mg1s, (e-h) Nb3d, and (i-1) Sn3d spectrum for the representative $\mathrm{Li}_{3} \mathrm{Mg}_{2-x / 3} \mathrm{Sn}_{x} \mathrm{Nb}_{1-2 x / 3} \mathrm{O}_{6}(x=0.1, x=0.4, x=0.8$, and $x=1.3)$ specimens.

Fig. S2 (a) SAED pattern for the $\mathrm{Li}_{3} \mathrm{Mg}_{2-x / 3} \mathrm{Sn}_{x} \mathrm{Nb}_{1-2 x / 3} \mathrm{O}_{6}(x=0.2)$ orthorhombic superstructures taken along [100] zone axis and the Miller indexes of the superlattice reflections. (b) Schematic delineation of the presumed reconstructed superstructures on (022) lattice planes observed along [100] zone axis for the orthorhombic phase.

Fig. S3 TEM-EDS (Transmission Electron Microscopy \& Energy Dispersive Spectroscopy) photographs for the $\mathrm{Li}_{3} \mathrm{Mg}_{2-x / 3} \mathrm{Sn}_{x} \mathrm{Nb}_{1-2 x / 3} \mathrm{O}_{6}(x=0,0.1,0.2$ and 0.8$)$ ceramics.

Fig. S4 SEM-EDS (Scanning Electron Microscopy \& Energy Dispersive Spectroscopy) images for $\mathrm{Li}_{3} \mathrm{Mg}_{2-x / 3} \mathrm{Sn}_{x} \mathrm{Nb}_{1-2 x / 3} \mathrm{O}_{6}(x=0.4$ and $x=0.8)$ ceramics $(\mathrm{a} \sim \mathrm{j})$, and element spectrum and weight percentage of atoms for $\mathrm{Li}_{3} \mathrm{Mg}_{2-x / 3} \mathrm{Sn}_{x} \mathrm{Nb}_{1-2 x / 3} \mathrm{O}_{6}(x=0.4$ and $x=1.4$ ) compounds ( $\mathrm{k}$ and $\mathrm{l}$ ). 
Fig. S5 Schematic delineation of $\mathrm{Nb}-\mathrm{Nb}$ vibration modes in $\mathrm{Nb}-\mathrm{Mg}-\mathrm{Nb}$ or $\mathrm{Nb}-\mathrm{Li}-\mathrm{Nb}$ clusters in the orthorhombic $\mathrm{Li}_{3} \mathrm{Mg}_{2-x / 3} \mathrm{Sn}_{x} \mathrm{Nb}_{1-2 x / 3} \mathrm{O}_{6}(0 \leq x \leq 0.6)$ structures.

Table S1 Bond length $\left(\mathrm{d}_{\mathrm{ij}}\right)$, bond valence parameters $\left(\mathrm{R}_{\mathrm{ij}}\right)$, the valence of chemical bonds $\left(\mathrm{v}_{\mathrm{ij}}\right)$, the observed valence of atoms $\left(\mathrm{V}_{\mathrm{ij}}\right)$, and global instability index (GII) of $\mathrm{Li}_{3} \mathrm{Mg}_{2-x / 3} \mathrm{Sn}_{x} \mathrm{Nb}_{1-2 x / 3} \mathrm{O}_{6}$ $(0 \leq x \leq 1.1)$ ceramics sintered at $1290^{\circ} \mathrm{C}$ for $4 \mathrm{~h}$.

\begin{tabular}{|c|c|c|c|c|c|c|c|}
\hline$x$ & Structure & Bond type & $\mathrm{d}_{\mathrm{ij}}(\AA)$ & $\mathrm{R}_{\mathrm{ij}}$ & $\mathrm{v}_{\mathrm{ij}}$ & $\mathrm{V}_{\mathrm{ij}}$ & GII (uv) \\
\hline \multirow[t]{18}{*}{0} & \multirow[t]{18}{*}{ Orthorhombic } & $\mathrm{Li}_{1}-\mathrm{O}_{1}$ & 2.09373 & 1.466 & 0.18331 & 1.09987 & 0.2484 \\
\hline & & $\mathrm{Li}_{2}-\mathrm{O}_{1}$ & 2.16771 & & 0.15009 & 0.90055 & \\
\hline & & $\mathrm{Li}_{3}-\mathrm{O}_{1}$ & 2.31558 & & 0.10064 & 0.60387 & \\
\hline & & \multirow{2}{*}{$\mathrm{Li}_{1}-\mathrm{O}_{2}$} & 2.19279 & & 0.14025 & 0.84153 & \\
\hline & & & 2.13825 & & 0.16253 & 0.97518 & \\
\hline & & \multirow{2}{*}{$\mathrm{Li}_{2}-\mathrm{O}_{2}$} & 2.09386 & & 0.18325 & 1.09948 & \\
\hline & & & 2.11256 & & 0.17422 & 1.04530 & \\
\hline & & $\mathrm{Li}_{3}-\mathrm{O}_{2}$ & 2.05002 & & 0.20630 & 1.23779 & \\
\hline & & $\mathrm{Mg}_{1}-\mathrm{O}_{1}$ & 2.09373 & 1.693 & 0.33856 & 2.03136 & \\
\hline & & $\mathrm{Mg}_{2}-\mathrm{O}_{1}$ & 2.16771 & & 0.27720 & 1.66323 & \\
\hline & & $\mathrm{Mg}_{3}-\mathrm{O}_{1}$ & 2.31558 & & 0.18588 & 1.11529 & \\
\hline & & \multirow{2}{*}{$\mathrm{Mg}_{1}-\mathrm{O}_{2}$} & 2.19279 & & 0.25904 & 1.55422 & \\
\hline & & & 2.13825 & & 0.30018 & 1.80107 & \\
\hline & & \multirow{2}{*}{$\mathrm{Mg}_{2}-\mathrm{O}_{2}$} & 2.09386 & & 0.33844 & 2.03065 & \\
\hline & & & 2.11256 & & 0.32176 & 1.93057 & \\
\hline & & $\mathrm{Mg}_{3}-\mathrm{O}_{2}$ & 2.05002 & & 0.38101 & 2.28608 & \\
\hline & & $\mathrm{Nb}_{1}-\mathrm{O}_{1}$ & 1.95973 & 1.911 & 0.87660 & 4.72896 & \\
\hline & & $\mathrm{Nb}_{1}-\mathrm{O}_{2}$ & 1.99908 & & 0.78816 & 5.25961 & \\
\hline \multirow[t]{17}{*}{0.1} & \multirow[t]{17}{*}{ Orthorhombic } & $\mathrm{Li}_{1}-\mathrm{O}_{1}$ & 2.10892 & 1.466 & 0.17594 & 1.05563 & 0.2745 \\
\hline & & $\mathrm{Li}_{2}-\mathrm{O}_{1}$ & 2.18475 & & 0.14334 & 0.86001 & \\
\hline & & $\mathrm{Li}_{3}-\mathrm{O}_{1}$ & 2.25381 & & 0.11893 & 0.71358 & \\
\hline & & \multirow{2}{*}{$\mathrm{Li}_{1}-\mathrm{O}_{2}$} & 2.18932 & & 0.14158 & 0.84946 & \\
\hline & & & 2.10797 & & 0.17639 & 1.05834 & \\
\hline & & \multirow{2}{*}{$\mathrm{Li}_{2}-\mathrm{O}_{2}$} & 2.10242 & & 0.17906 & 1.07434 & \\
\hline & & & 2.08862 & & 0.18586 & 1.11517 & \\
\hline & & $\mathrm{Li}_{3}-\mathrm{O}_{2}$ & 2.06629 & & 0.19742 & 1.18454 & \\
\hline & & $\mathrm{Mg}_{1}-\mathrm{O}_{1}$ & 2.09373 & 1.693 & 0.32494 & 1.94965 & \\
\hline & & $\mathrm{Mg}_{2}-\mathrm{O}_{1}$ & 2.16771 & & 0.26473 & 1.58837 & \\
\hline & & $\mathrm{Mg}_{3}-\mathrm{O}_{1}$ & 2.31558 & & 0.21965 & 1.31792 & \\
\hline & & \multirow{2}{*}{$\mathrm{Mg}_{1}-\mathrm{O}_{2}$} & 2.18932 & & 0.26148 & 1.56887 & \\
\hline & & & 2.10797 & & 0.32578 & 1.95467 & \\
\hline & & \multirow{2}{*}{$\mathrm{Mg}_{2}-\mathrm{O}_{2}$} & 2.10242 & & 0.33070 & 1.98421 & \\
\hline & & & 2.08862 & & 0.34327 & 2.05961 & \\
\hline & & $\mathrm{Mg}_{3}-\mathrm{O}_{2}$ & 2.06629 & & 0.36462 & 2.18774 & \\
\hline & & $\mathrm{Nb}_{1}-\mathrm{O}_{1}$ & 2.02523 & 1.911 & 0.73438 & 4.40628 & \\
\hline
\end{tabular}




\begin{tabular}{|c|c|c|c|c|c|c|c|}
\hline & & $\mathrm{Nb}_{1}-\mathrm{O}_{2}$ & 2.00968 & & 0.76590 & 4.59540 & \\
\hline $0.2-0.7$ & Multi-phase & & & & & & \\
\hline \multirow[t]{4}{*}{0.8} & Cubic & Li-O & 2.12127 & 1.466 & 0.17016 & 1.02098 & 0.7319 \\
\hline & & $\mathrm{Mg}-\mathrm{O}$ & 2.12127 & 1.693 & 0.31428 & 1.88565 & \\
\hline & & $\mathrm{Nb}-\mathrm{O}$ & 2.12127 & 1.911 & 0.56649 & 3.39894 & \\
\hline & & Sn-O & 2.12127 & 1.905 & 0.55738 & 3.34426 & \\
\hline \multirow[t]{4}{*}{0.9} & Cubic & Li-O & 2.12369 & 1.466 & 0.16905 & 1.01432 & 0.7390 \\
\hline & & $\mathrm{Mg}-\mathrm{O}$ & 2.12369 & 1.693 & 0.31223 & 1.87336 & \\
\hline & & $\mathrm{Nb}-\mathrm{O}$ & 2.12369 & 1.911 & 0.56280 & 3.37678 & \\
\hline & & Sn-O & 2.12369 & 1.905 & 0.55374 & 3.32246 & \\
\hline \multirow[t]{4}{*}{1.0} & Cubic & Li-O & 2.12489 & 1.466 & 0.16851 & 1.01104 & 0.7469 \\
\hline & & $\mathrm{Mg}-\mathrm{O}$ & 2.12489 & 1.693 & 0.31122 & 1.86729 & \\
\hline & & $\mathrm{Nb}-\mathrm{O}$ & 2.12489 & 1.911 & 0.56097 & 3.36584 & \\
\hline & & Sn-O & 2.12489 & 1.905 & 0.55195 & 3.31170 & \\
\hline \multirow[t]{4}{*}{1.1} & Cubic & Li-O & 2.12828 & 1.466 & 0.16697 & 1.00182 & 0.7530 \\
\hline & & $\mathrm{Mg}-\mathrm{O}$ & 2.12828 & 1.693 & 0.30838 & 1.85026 & \\
\hline & & $\mathrm{Nb}-\mathrm{O}$ & 2.12828 & 1.911 & 0.55586 & 3.33515 & \\
\hline & & $\mathrm{Sn}-\mathrm{O}$ & 2.12828 & 1.905 & 0.54692 & 3.28150 & \\
\hline
\end{tabular}


Fig. S1 XPS core-level (a-d) Mg1s, $(\mathrm{e}-\mathrm{h}) \mathrm{Nb3d}$, and (i-1) Sn3d spectrum for the representative $\mathrm{Li}_{3} \mathrm{Mg}_{2-x / 3} \mathrm{Sn}_{x} \mathrm{Nb}_{1-2 x / 3} \mathrm{O}_{6}(x=0.1, x=0.4, x=0.8$, and $x=1.3)$ specimens. 

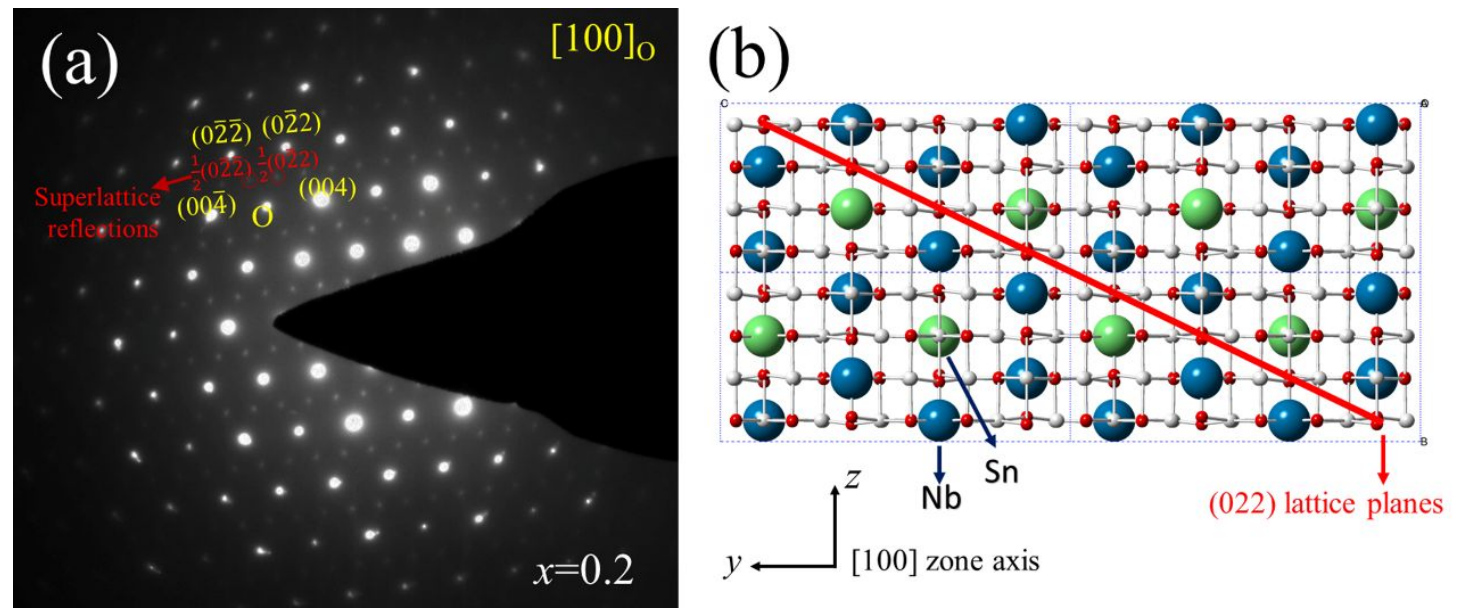

Fig. S2 (a) SAED pattern for the $\mathrm{Li}_{3} \mathrm{Mg}_{2-x / 3} \mathrm{Sn}_{x} \mathrm{Nb}_{1-2 x / 3} \mathrm{O}_{6}(x=0.2)$ orthorhombic superstructures taken along [100] zone axis and the Miller indexes of the superlattice reflections. (b) Schematic delineation of the presumed reconstructed superstructures on (022) lattice planes observed along [100] zone axis for the orthorhombic phase. 


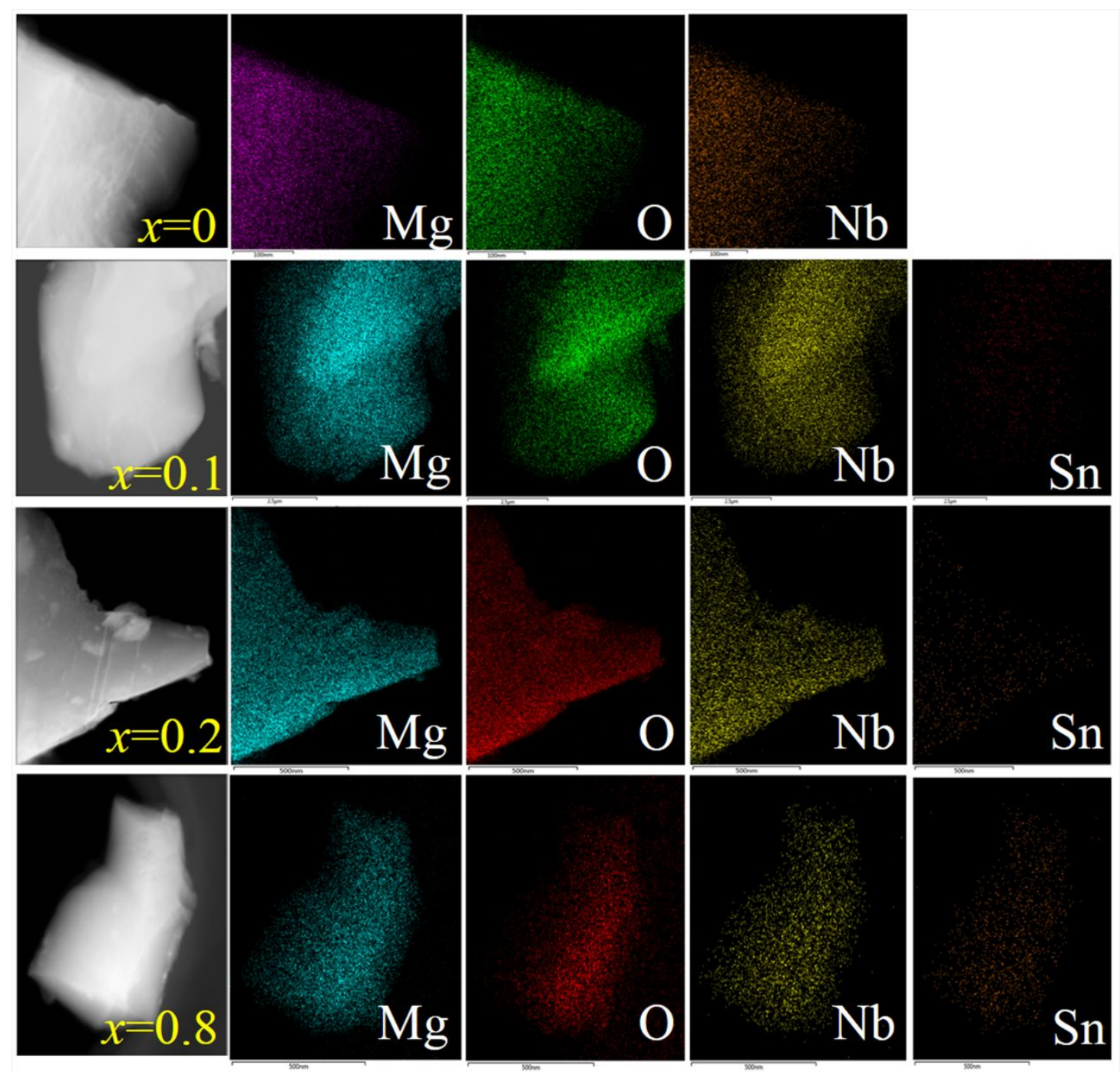

Fig. S3 TEM-EDS (Transmission Electron Microscopy \& Energy Dispersive Spectroscopy) photographs for the $\mathrm{Li}_{3} \mathrm{Mg}_{2-x / 3} \mathrm{Sn}_{x} \mathrm{Nb}_{1-2 x / 3} \mathrm{O}_{6}(x=0,0.1,0.2$ and 0.8$)$ ceramics. 


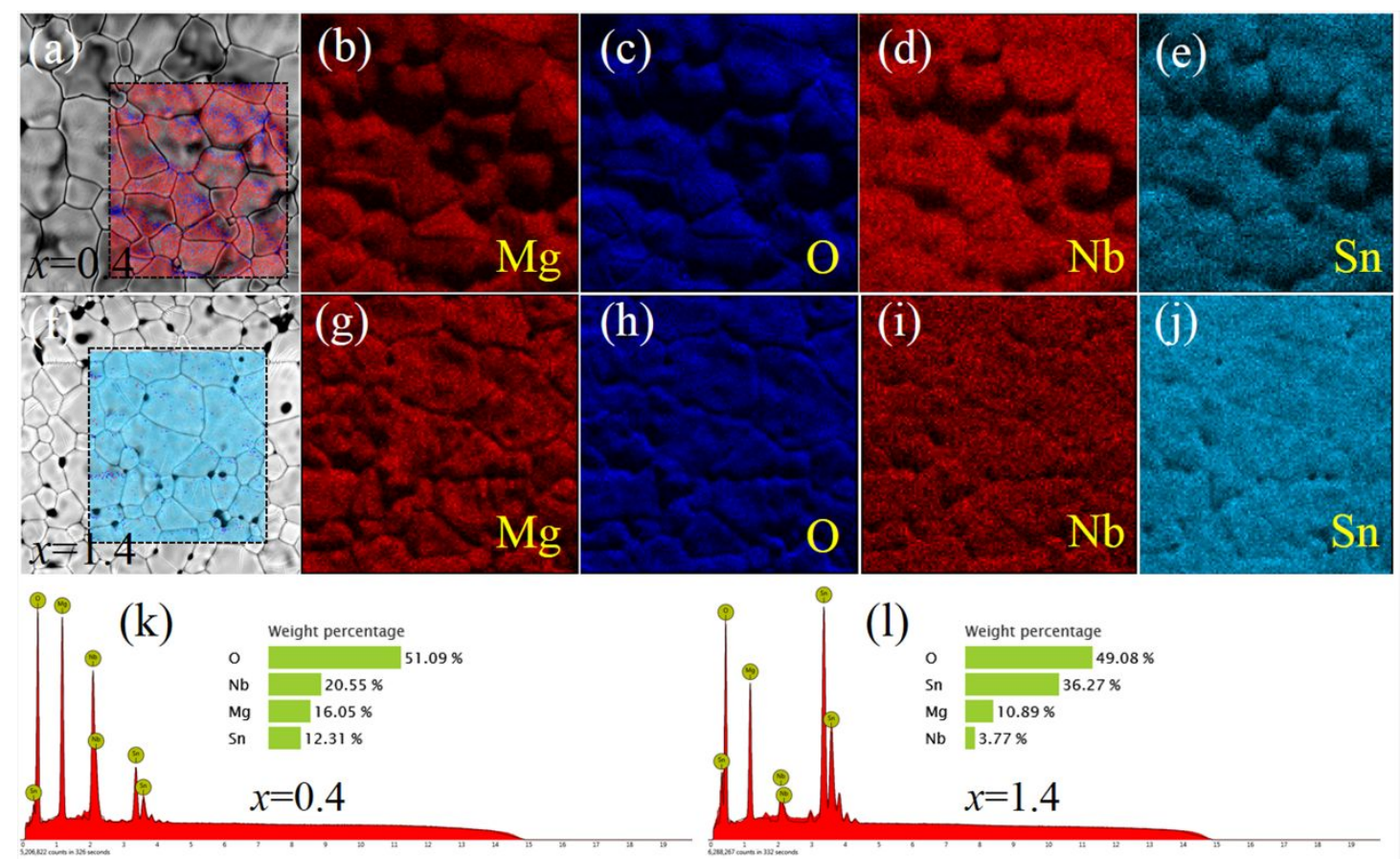

Fig. S4 SEM-EDS (Scanning Electron Microscopy \& Energy Dispersive Spectroscopy) images for $\mathrm{Li}_{3} \mathrm{Mg}_{2-x / 3} \mathrm{Sn}_{x} \mathrm{Nb}_{1-2 x / 3} \mathrm{O}_{6}(x=0.4$ and $x=0.8)$ ceramics $(\mathrm{a}-\mathrm{j})$, and element spectrum and weight percentage of atoms for $\mathrm{Li}_{3} \mathrm{Mg}_{2-x / 3} \mathrm{Sn}_{x} \mathrm{Nb}_{1-2 x / 3} \mathrm{O}_{6}(x=0.4$ and $x=1.4)$ compounds $(\mathrm{k}$ and $\mathrm{l})$.

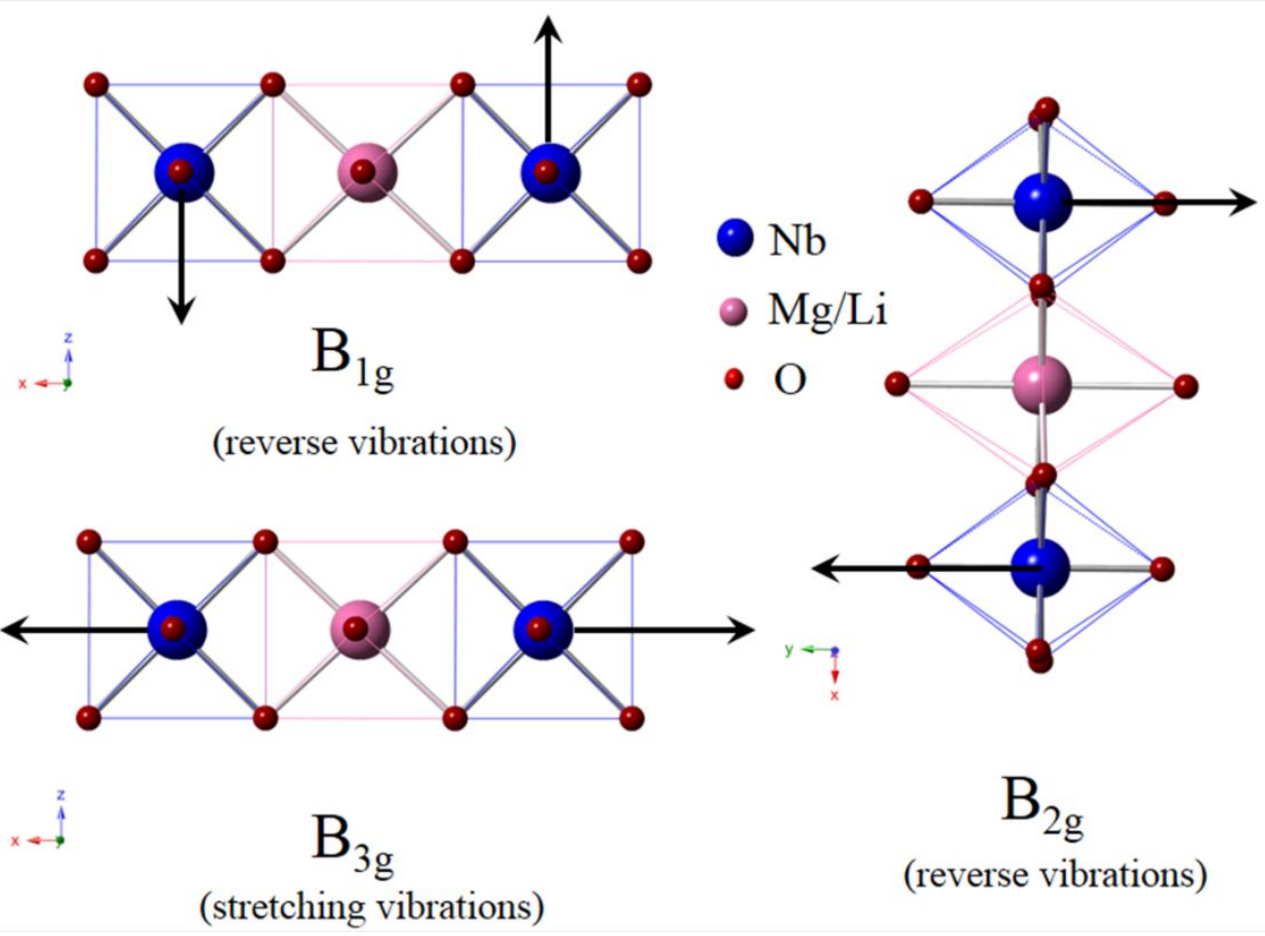

Fig. S5 Schematic delineation of $\mathrm{Nb}-\mathrm{Nb}$ vibration modes in $\mathrm{Nb}-\mathrm{Mg}-\mathrm{Nb}$ or $\mathrm{Nb}-\mathrm{Li}-\mathrm{Nb}$ clusters in the orthorhombic $\mathrm{Li}_{3} \mathrm{Mg}_{2-x / 3} \mathrm{Sn}_{x} \mathrm{Nb}_{1-2 x / 3} \mathrm{O}_{6}(0 \leqslant x \leqslant 0.6)$ structures. 